\title{
Comparación teórica-numérica de la sobrepresión generada en sistemas de tuberías de diámetro variable
}

\section{Theoretical-numerical comparison of water hammer generated in piping systems with variable diameter}

Fecha de entrega: 2 de diciembre 2019

Fecha de aceptación: 19 de marzo 2020

\section{Iván Salazar y Alen Kong}

Departamento de Ingeniería Civil, Universidad Católica del Norte, Avda. Angamos 0610, Antofagasta, Chile, isalazar@ucn.cl, akong@ucn.cl

Se analiza y compara el valor de la sobrepresión en una válvula ubicada aguas abajo en un sistema por gravedad, cuando se presentan variaciones en las propiedades geométricas en la conducción (cambios de diámetro). Se analiza la validez de los resultados obtenidos comparando dos metodologías, a saber: modelación numérica por elementos finitos y la aplicación de expresiones teóricas basadas en el método de celeridad y longitud equivalente. Para la resolución numérica de las ecuaciones se desarrolla una rutina computacional en Matlab que permite representar la propagación de las ondas de presión en la tubería. Se define el modelo numérico considerando todas las condiciones que presenta el problema y se examinan distintos casos en los que se presenten variaciones en las propiedades geométricas en la conducción considerando ensanches y angostamientos en distintas ubicaciones a lo largo de la conducción y se comparan con los resultados de las presiones máximas obtenidos mediante las aproximaciones teóricas.

Palabras claves: golpe de ariete, movimentos transitorios en conductos a presion, método de los elementos finitos
This study analyses and compares the value of the overpressure in a valve located downstream in a gravity system when there are variations in the geometric properties in the conduction (diameter changes). In addition, the validity of the results obtained is analysed by comparing two methodologies: numerical modelling by finite elements and the application of theoretical expressions based on the method of celerity and equivalent length. For the numerical resolution of the equations a computational routine is developed in Matlab that allows representing the propagation of the pressure waves in the pipe. Then, the numerical model is defined considering all the conditions that the problem presents, and different cases are examined in which their variations in the geometric properties in the conduction are considering widenings and narrowing in different locations throughout the conduction. Finally, the study makes a comparison with the results of the maximum pressures obtained through the theoretical approximations.

Keywords: water hammer, transient movements in pipes under pressure, finite element method

\section{Introducción}

En el análisis de movimentos transitorios en conductos a presión se estudian las presiones y velocidades que resultan del cambio desde un estado permanente a otro no permanente, comúnmente denominadas perturbaciones hidráulicas. Dichas perturbaciones ocurren cuando el fluido conducido por una tubería en presión se ve obligado a variar su velocidad o dirección repentinamente. Esto provoca una secuencia de sobrepresiones y subpresiones, las cuales deforman las tuberías y eventualmente ocasionan rompimiento de piezas y conductos (Streeter y Wylie, 1999). Este fenómeno se produce comúnmente cuando se realizan maniobras de apertura o cierre de válvulas, o también al poner en marcha o detener una máquina hidráulica.

Las ecuaciones de Saint Venant son las que rigen los movimientos transitorios en conducciones en presión y vienen dadas por (Parmakian, 1963; Streeter y Wylie, 1999; Bollrich, 2007): 


$$
\begin{aligned}
& \frac{\partial v}{\partial t}+\mathrm{g} \frac{\partial H}{\partial x}+\mathrm{g} \operatorname{sen} \theta+f \frac{v|v|}{2 D}=0 \\
& \mathrm{~g} \frac{\partial H}{\partial \mathrm{t}}+\mathrm{c}^{2} \frac{\partial \mathrm{v}}{\partial \mathrm{x}}=0
\end{aligned}
$$

donde $v(\mathrm{~L} / \mathrm{T})$ es la velocidad media del flujo en cada sección y en cada instante, $H(\mathrm{~L})$ representa la energía contenida en el fluido en cada sección y en cada instante, $g$ $\left(\mathrm{L} / \mathrm{T}^{2}\right)$ es la aceleración de gravedad, $\operatorname{sen} \theta$ es la pendiente del conducto, $f(-)$ representa el factor de fricción del conducto, $D(\mathrm{~L})$ representa el diámetro interno de la tubería y $c(\mathrm{~L} / \mathrm{T})$ corresponde a la celeridad de la onda.

Las ecuaciones (1) y (2) representan un sistema de ecuaciones diferenciales parciales no lineales. El término c en (2) representa a la celeridad o velocidad de propagación de la onda de presión a través del líquido. La celeridad viene dada por (Parmakian, 1963; Twyman, 2016):

$$
\mathrm{c}=\sqrt{\frac{1}{\rho\left(\frac{1}{K}+\frac{D c_{1}}{e E}\right)}}
$$

donde $\rho\left(\mathrm{M} / \mathrm{L}^{3}\right)$ es la densidad del fluido, $K\left(\mathrm{M} / \mathrm{T}^{2} \mathrm{~L}\right)$ corresponde al módulo de compresibilidad del líquido, $D$ $(\mathrm{L})$ es el diámetro interno del conducto, $e(\mathrm{~L})$ representa el espesor del conducto, $E\left(\mathrm{M} / \mathrm{T}^{2} \mathrm{~L}\right)$ es el módulo de elasticidad del material del conducto y $\mathrm{C}_{1}$ (-) es una constante que representa el tipo de unión que posee el conducto.

Para el caso de evaluación de la sobrepresión producido por el cierre de una válvula en un sistema gravitacional, se introduce el concepto de tiempo crítico, el cual diferencia los casos de cierres bruscos con los cierres lentos (Allievi, 1903; Parmakian, 1963; Bollrich, 2007):

$$
t_{c}=\frac{2 L}{c}
$$

donde $L(\mathrm{~L})$ es la longitud del conducto y $c(\mathrm{~L} / \mathrm{T})$ es la celeridad de la onda. Se considera cierre brusco si el tiempo de cierre de la válvula $t_{\mathrm{cv}}$ es menor al tiempo crítico, por el contrario, se considera cierre lento si el tiempo de cierre de la válvula $t_{\mathrm{cv}}$ es mayor al tiempo crítico. De acuerdo al tipo de cierre se utilizan distintas expresiones para determinar los valores de sobrepresión en el conducto. Para cierres bruscos se utiliza la expresión (5) de Joukowsky, mientras que para cierres suave se utiliza la expresión (6) de Michaud (Parmakian, 1963; Bollrich, 2007).

$$
\begin{aligned}
& \Delta h_{\max }=\frac{c v}{\mathrm{~g}} \\
& \Delta h_{\max }=\frac{2 L v}{\mathrm{~g} t_{\mathrm{cv}}}
\end{aligned}
$$

Cuando existen conducciones que presentan cambios en sus propiedades geométricas y/o elásticas es posible obtener una solución satisfactoria a través de la obtención de una tubería uniforme equivalente (Parmakian, 1963), la cual relaciona los largos, áreas y celeridades de los distintos tramos para determinar una tubería de condición equivalente, a saber:

$$
\begin{aligned}
& \frac{L_{\mathrm{eq}}}{A}=\left(\frac{L_{1}}{A_{1}}+\frac{L_{2}}{A_{2}}+\cdots+\frac{L_{\mathrm{n}}}{A_{\mathrm{n}}}\right)=\sum_{i=1}^{n}\left(\frac{L_{\mathrm{i}}}{A_{\mathrm{i}}}\right) \\
& \frac{L}{c}=\left(\frac{L_{1}}{c_{1}}+\frac{L_{2}}{c_{2}}+\cdots+\frac{L_{\mathrm{n}}}{c_{\mathrm{n}}}\right)=\sum_{i=1}^{n}\left(\frac{L_{\mathrm{i}}}{c_{\mathrm{i}}}\right)
\end{aligned}
$$

donde las variables con subíndices corresponde a $\operatorname{los} n$ tramos con características homogéneas, tanto de longitud, área y celeridad.

Es importante notar que (7) hace referencia a la obtención de una longitud equivalente válida sólo bajo las condiciones de columna rígida, es decir, considerando flujo ideal y conducto rígido (Parmakian, 1963; Bollrich, 2007). Por otra parte, (8) hace referencia a obtener una celeridad equivalente bajo la teoría de columna flexible (Parmakian, 1963). Sin embargo, para una conducción en serie, en (8) no se diferencia sobre el tipo de cambio de geometría. Se trata indistintamente un ensanche como un angostamiento. Tampoco se hace mención a la ubicación del cambio de geometría, es decir, si dicho ensanche o angostamiento se encuentra justo en la mitad de la longitud del conducto o a una distancia mayor o a una distancia menor, no parece tener efecto en dicha ecuación (Kong y Salazar, 2017). Un análisis detallado sobre conducciones en régimen transitorios se puede encontrar, por ejemplo, en Parmakian (1963), Abreu et al. (1995) y en Bollrich (2007) y estudios de casos particulares, por ejemplo, en Twyman (2018, 2019). 


\section{Modelo numérico}

Se desarrolla un modelo numérico basado en los esquemas indicados en la Figura 1. Se utiliza el método de los elementos finitos (e.g. Zienkiewicz y Taylor, 2000) para obtener el valor de la sobrepresión en una válvula (obturador) ubicada aguas abajo en un sistema gravitacional. Se comparan los resultados obtenidos con los calculados mediante la aproximación teórica basado en el cálculo de una longitud y celeridad equivalente.

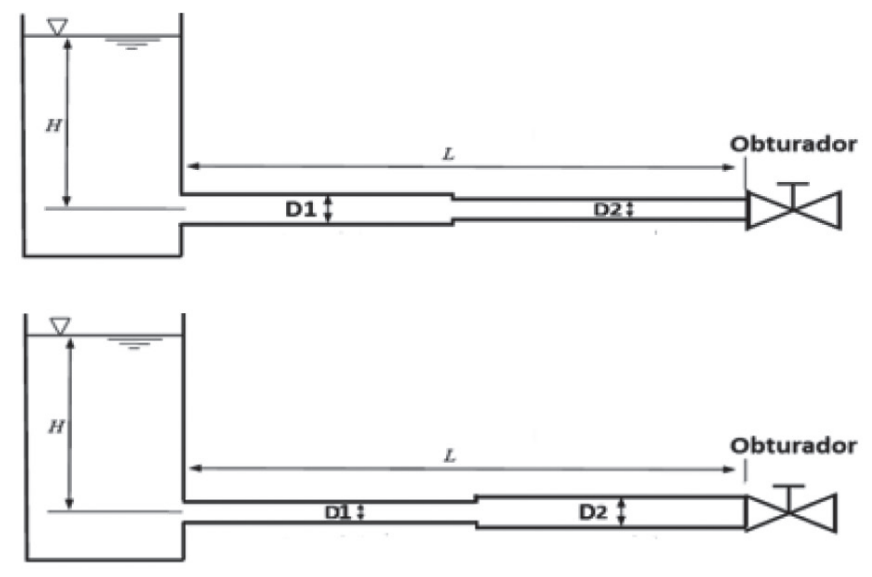

Figura 1: Modelos de análisis

Las características del modelo de análisis de la Figura 1 son las siguientes:

- Estanque de altura constante $10 \mathrm{~m}$ conectado a un sistema de tuberías funcionando gravitacionalmente con una válvula de control a la salida.

- Se considera un sistema de tuberías de $1000 \mathrm{~m}$ de largo conformado por dos tramos en serie, los cuales presentan variaciones de diámetro (ensanche/angostamiento), pero con el material de la tubería constante.

- La condición inicial es el momento cuando el sistema se encuentra en régimen permanente y se realiza el cierre de la válvula de control.

- El cierre de la válvula de control corresponde a un cierre brusco.

- El tiempo de simulación es de $t=50 \mathrm{~s}$ con un intervalo de tiempo $\Delta t=0.02 \mathrm{~s}$.

Las ecuaciones (1) y (2) se trabajan mediante el método de los elementos finitos. Se discretiza el dominio en elementos finitos lineales de longitud $\Delta L$ constante para cada tramo, los cuales están definidos por sus propiedades físicas y se encuentran unidos por nodos en sus extremos según se muestra en la Figura 2.

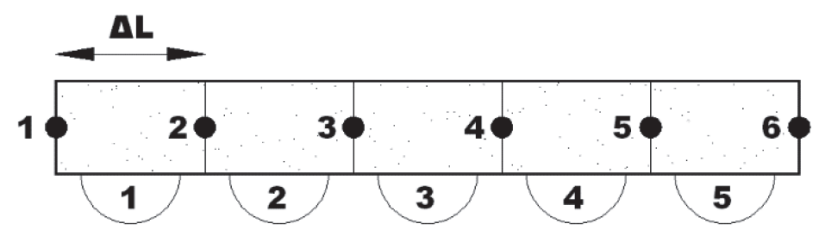

Figura 2: Discretización lineal de elementos finitos

La discretización espacial se realiza por medio del método del desplazamiento virtual (Videla y Salazar, 2010; Salazar et al., 2011). De este modo, (1) y (2) para cada elemento, se expresan de la siguiente forma:

$\frac{1}{3}\left[\begin{array}{cc}\Delta L & \frac{\Delta L}{2} \\ \frac{\Delta L}{2} & \Delta L\end{array}\right]\left[\begin{array}{c}\dot{v}^{\mathrm{e} 1} \\ \dot{v}^{\mathrm{e}} \mathrm{e}\end{array}\right]+\frac{\mathrm{g}}{2}\left[\begin{array}{cc}-1 & 1 \\ -1 & 1\end{array}\right]\left[\begin{array}{c}H^{\mathrm{e} 1} \\ H^{\mathrm{e} 2}\end{array}\right]+\frac{\mathrm{f}|v|}{2 D}\left[\begin{array}{cc}\Delta L & \frac{\Delta L}{2} \\ \frac{\Delta L}{2} & \Delta L\end{array}\right]\left[\begin{array}{l}v^{\mathrm{e} 1} \\ v^{\mathrm{e}}\end{array}\right]=0$

$\frac{\mathrm{g}}{3}\left[\begin{array}{cc}\Delta L & \frac{\Delta L}{2} \\ \frac{\Delta L}{2} & \Delta L\end{array}\right]\left[\begin{array}{l}\dot{H}^{\mathrm{e} 1} \\ \dot{H}^{\mathrm{e} 2}\end{array}\right]+\frac{c^{2}}{2}\left[\begin{array}{ll}-1 & 1 \\ -1 & 1\end{array}\right]\left[\begin{array}{l}v^{\mathrm{e} 1} \\ v^{\mathrm{e} 2}\end{array}\right]=0$

Ensamblando las ecuaciones de todos los elementos, se obtienen las matrices globales del sistema de ecuaciones que rigen el comportamiento del golpe de ariete en un sistema de tuberías:

$$
\begin{aligned}
& \mathrm{A} \dot{\mathrm{v}}+\mathrm{BH}+\mathrm{Cv}=0 \\
& \mathrm{DH}+\mathrm{Ev}=0
\end{aligned}
$$

El sistema se expresa en base a las propiedades físicas conocidas de los elementos de tuberías, mientras que las incógnitas son los vectores correspondientes a los valores de velocidad y presión en cada nodo de la tubería y su variación temporal. Se utiliza el método alfa generalizado (Hughes, 1987; Chung y Hulbert, 1993; Jansen et al., 2000) para la discretización temporal de las ecuaciones (9) y (10). Desarrollando las ecuaciones del método alfa generalizado se tiene:

$\Delta v\left(\frac{A\left(1-\alpha_{\mathrm{m}}\right)}{\gamma \Delta t}+C\left(1-\alpha_{\mathrm{f}}\right)\right)+\Delta H B\left(1-\alpha_{\mathrm{f}}\right)=-A\left(\dot{v}_{\mathrm{n}}\left(\frac{\gamma-1}{\gamma}\right)\left(1-\alpha_{\mathrm{m}}\right)+\dot{v}_{\mathrm{n}} \alpha_{\mathrm{m}}\right)-B H_{\mathrm{n}}-C v_{\mathrm{n}}$

$\Delta H\left(\frac{D\left(1-\alpha_{\mathrm{m}}\right)}{\gamma \Delta t}\right)+\Delta v E\left(1-\alpha_{\mathrm{f}}\right)=-D\left(\dot{H}_{\mathrm{n}}\left(\frac{\gamma-1}{\gamma}\right)\left(1-\alpha_{\mathrm{m}}\right)+\dot{H}_{\mathrm{n}} \alpha_{\mathrm{m}}\right)-E v_{\mathrm{n}}$

El sistema de ecuaciones (13) y (14) se expresa en función de parámetros temporales del método alfa, matrices y vectores dependientes de las características físicas de las tuberías y de vectores de variación temporal de velocidad 
y presión respectivamente. Además, para el tiempo siguiente $t=n+1$ se tiene:

$$
\begin{aligned}
& H_{\mathrm{n}+1}=H_{\mathrm{n}}+\Delta H \\
& \dot{H}_{\mathrm{n}+1}=\frac{\Delta H}{\gamma \Delta t}+\dot{H}_{\mathrm{n}}\left(\frac{\gamma-1}{\gamma}\right)
\end{aligned}
$$

Se define el radio espectral de la matriz, además de los parámetros del método alfa $\left(\alpha_{f}=0.5128, \alpha_{m}=0.5256\right.$ y $\gamma=$ 0.5128 ) de tal manera que aseguren la convergencia del método (Chung y Hulbert, 1993; Jansen et al., 2000). Los valores de velocidad y presión en los nodos de cada elemento se determinan resolviendo el sistema de ecuaciones resultantes. Las condiciones de borde del problema en este caso es el nivel constante del estanque y el cierre brusco de la válvula de control (ambos para $t=0$ ).

\section{Casos simulados}

El modelo de análisis es una conducción compuesta por dos tramos de igual material, pero de distinto diámetro. El cambio de diámetro se evalúa en distintos puntos de la conducción para ver su incidencia en los valores de máxima sobrepresión obtenidos. Las características hidráulicas para el modelo numérico se muestran en la Tabla 1.

Tabla 1: Características definidas para el modelo numérico

\begin{tabular}{|l|c|}
\hline Tramos de tubería & 2 \\
\hline Altura de líquido en el estanque, $\mathrm{m}$ & 10 \\
\hline Longitud, $\mathrm{m}$ & 1000 \\
\hline Material & HDPE \\
\hline Tiempo de simulación, $\mathrm{s}$ & 50 \\
\hline
\end{tabular}

Para validar el modelo a través del método de los elementos finitos se determina y compara el valor de la máxima sobrepresión obtenida en la válvula de control en metros de columna de agua mca, utilizando la expresión analítica con aproximación de celeridad equivalente. Los datos considerados son los mostrados en la Tabla 1 considerando un conducto de HDPE PN10 de un solo tramo de diámetro nominal $D=160$ $\mathrm{mm}$ (6"), de espesor $e=0.015 \mathrm{~m}$ y de rugosidad absoluta $\varepsilon=0.0015 \mathrm{~mm}$. Los resultados se indican en la Tabla 2 .

Se analizan 2 casos en particular, conexión de tubería en serie con ensanche y angostamiento. Se comparan los resultados obtenidos a través de la modelación numérica con los obtenidos por la aproximación de tubería de longitud equivalente. La comparación se enfoca en el comportamiento de la onda de presión, en el periodo de onda y en los valores máximos de presión en la válvula de control.

Tabla 2: Máxima sobrepresión obtenida en la válvula del sistema

\begin{tabular}{|l|c|}
\hline Método & Máxima sobrepresión \\
\hline Expresión de Joukowsky, mca & 110.98 \\
\hline Método de los elementos finitos, mca & 109.59 \\
\hline
\end{tabular}

mca: metros de columna de agua

\section{Caso ensanche}

Se consideran dos casos de ensanches, a saber: ensanche de tubería de diámetro nominal $D=50.8 \mathrm{~mm}$ (2") a otra de $D=63.5\left(2.5^{\prime \prime}\right)$ y ensanche de diámetro nominal $D=$ 50.8 (2") a otra de $D=101.6 \mathrm{~mm}$ (4"). Para cada ensanche considerado en la tubería se analizan tres casos de análisis, los cuales se diferencian por el lugar en el que se produce el cambio de tramo. En el primer caso el ensanche ocurre a $200 \mathrm{~m}$ del estanque, en el segundo caso a $500 \mathrm{~m}$ del estanque y el tercer caso a $800 \mathrm{~m}$ del estanque. En la Tabla 3 se muestran las características y resultados obtenidos de los tres sistemas que se analizan para el caso de ensanche de tubería de 2" a 2.5", mientras que en la Tabla 4 se muestran las características y los resultados obtenidos aplicando el concepto de celeridad y longitud equivalente.

Tabla 3: Características definidas y valor de máxima sobrepresión obtenida para el caso de ensanche de tubería de 2" a 2.5" a través de la modelación numérica por elementos finitos.

\begin{tabular}{|l|c|c|c|c|c|c|}
\hline $\begin{array}{l}\text { Sistema de } \\
\text { tuberías }\end{array}$ & \multicolumn{2}{|c|}{ A } & \multicolumn{2}{c|}{ B } & \multicolumn{2}{c|}{ C } \\
\hline Tramo & 1 & 2 & 1 & 2 & 1 & 2 \\
\hline Longitud, m & 200 & 800 & 500 & 500 & 800 & 200 \\
\hline Diámetro, m & 0.049 & 0.058 & 0.049 & 0.058 & 0.049 & 0.058 \\
\hline Material & HDPE & HDPE & HDPE & HDPE & HDPE & HDPE \\
\hline Celeridad, m/s & 604.78 & 604.66 & 604.78 & 604.66 & 604.78 & 604.66 \\
\hline $\begin{array}{l}\text { Velocidad } \\
\text { inicial, m/s }\end{array}$ & 1.36 & 0.97 & 1.18 & 0.84 & 1.06 & 0.76 \\
\hline Caudal, m 3 /s & 0.0026 & 0.0022 & 0.0020 \\
\hline $\begin{array}{l}\text { Máxima } \\
\text { sobrepresión, } \\
\text { mca }\end{array}$ & \multicolumn{7}{|c|}{87.42} & \multicolumn{2}{|c|}{78.85} & 73.28 \\
\hline
\end{tabular}

Tabla 4: Características definidas y valor de máxima sobrepresión obtenida para el caso de ensanche de tubería de 2" a 2.5" a través del método de celeridad y longitud equivalente

\begin{tabular}{|l|c|c|c|}
\hline Sistema de tuberías & $\mathrm{A}$ & $\mathrm{B}$ & $\mathrm{C}$ \\
\hline Longitud equivalente, $\mathrm{m}$ & 1080 & 1200 & 1320 \\
\hline Diámetro equivalente, $\mathrm{m}$ & 0.058 & 0.058 & 0.058 \\
\hline Celeridad equivalente, $\mathrm{m} / \mathrm{s}$ & 653.06 & 725.66 & 798.27 \\
\hline Velocidad inicial, $\mathrm{m} / \mathrm{s}$ & 0.97 & 0,84 & 0.76 \\
\hline Máxima sobrepresión, mca & 73.34 & 71.15 & 70.28 \\
\hline
\end{tabular}


Las Figuras 3 a 8 muestran la comparación de los valores de la sobrepresión en función del tiempo en la válvula de control, obtenidos a través de la modelación numérica por elementos finitos y por el método de celeridad y longitud equivalente a distintas ubicaciones del cambio de geometría y para relaciones de cambio de ensanche $D_{1} / D_{2}$ $=2 " / 2.5 "$ y $D_{1} / D_{2}=2 " / 4 "$.

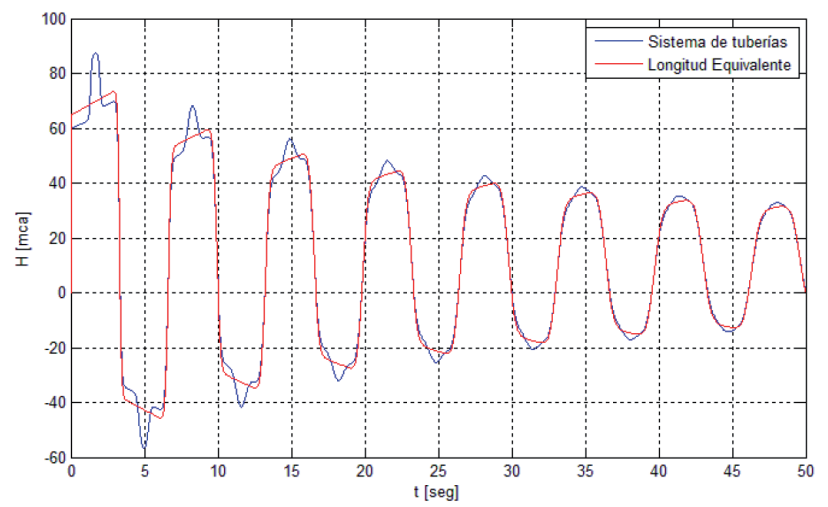

Figura 3: Variación de la sobrepresión en la válvula de control del sistema para ensanche $D_{1} / D_{2}=2 " / 2.5 "$ con ubicación de ensanche a una distancia $L_{1}=200 \mathrm{~m}$

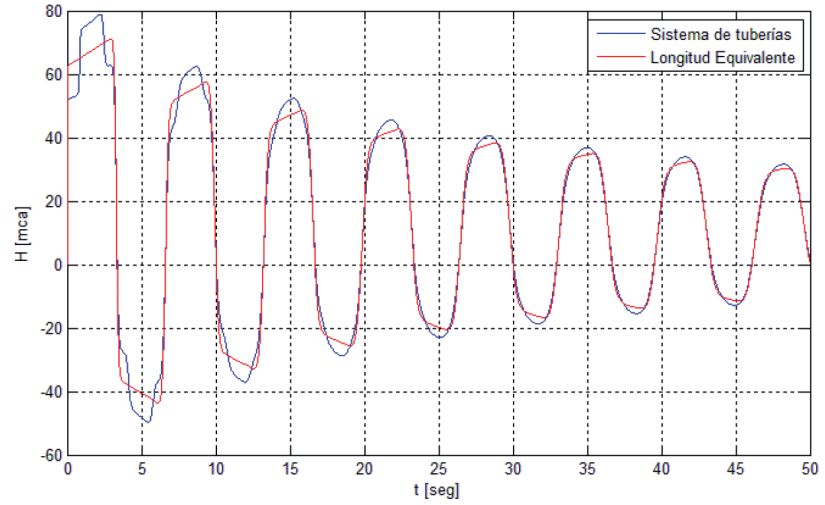

Figura 4: Variación de la sobrepresión en la válvula de control del sistema para ensanche $D_{1} / D_{2}=2 " / 2.5 "$ con ubicación de ensanche a una distancia $L_{1}=500 \mathrm{~m}$

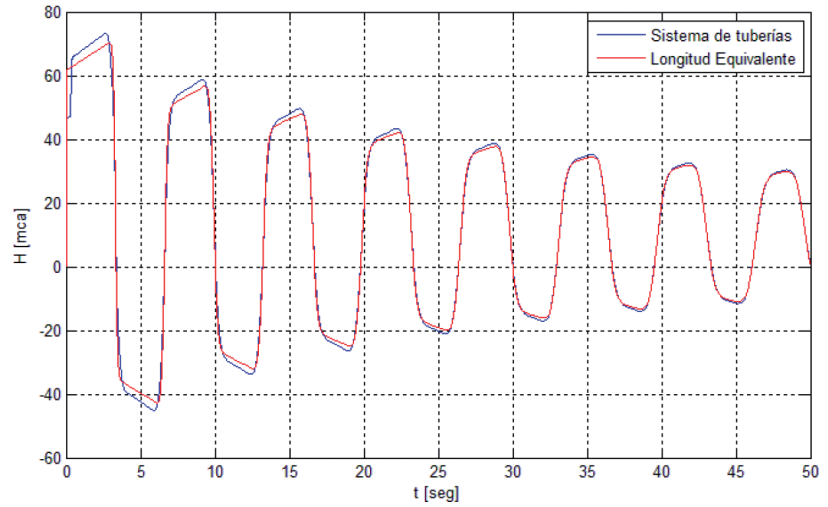

Figura 5: Variación de la sobrepresión en la válvula de control del sistema para ensanche $D_{1} / D_{2}=2 " / 2.5 "$ con ubicación de ensanche a una distancia $L_{1}=800 \mathrm{~m}$
En la Tabla 5 se muestran las características y resultados obtenidos de los tres sistemas que se analizan para el caso de ensanche de tubería de 2" a 4", mientras que en la Tabla 6 se indican las características y los resultados obtenidos del sistema aplicando el concepto de celeridad y longitud equivalente.

Tabla 5: Características definidas y valor de máxima sobrepresión obtenida para el caso de ensanche de tubería de 2" a 4" a través de la modelación numérica por elementos finitos

\begin{tabular}{|l|c|c|c|c|c|c|}
\hline $\begin{array}{l}\text { Sistema de } \\
\text { tuberías }\end{array}$ & \multicolumn{2}{|c|}{ A } & \multicolumn{2}{c|}{ B } & \multicolumn{2}{c|}{ C } \\
\hline Tramo & 1 & 2 & 1 & 2 & 1 & 2 \\
\hline Longitud, m & 200 & 800 & 500 & 500 & 800 & 200 \\
\hline Diámetro, m & 0.049 & 0.085 & 0.049 & 0.085 & 0.049 & 0.085 \\
\hline Material & HDPE & HDPE & HDPE & HDPE & HDPE & HDPE \\
\hline Celeridad, m/s & 604.78 & 604.45 & 604.78 & 604.45 & 604.78 & 604.45 \\
\hline $\begin{array}{l}\text { Velocidad } \\
\text { inicial, m/s }\end{array}$ & 2.00 & 0.66 & 1.37 & 0.45 & 1.10 & 0.37 \\
\hline Caudal, m $3 / \mathrm{s}$ & 0.0038 & 0.0026 & 0.0021 \\
\hline $\begin{array}{l}\text { Máxima } \\
\text { sobrepresión, } \\
\text { mca }\end{array}$ & \multicolumn{2}{|c|}{123.94} & \multicolumn{7}{|c|}{89.66} & 75.99 \\
\hline
\end{tabular}

Tabla 6: Características definidas y valor de máxima sobrepresión obtenida para el caso de ensanche de tubería de 2" a 4" a través del método de celeridad y longitud equivalente.

\begin{tabular}{|l|c|c|c|}
\hline Sistema de tuberías & $\mathrm{A}$ & $\mathrm{B}$ & $\mathrm{C}$ \\
\hline Longitud equivalente, $\mathrm{m}$ & 1400 & 2000 & 2610 \\
\hline Diámetro equivalente, $\mathrm{m}$ & 0.085 & 0.085 & 0.085 \\
\hline Celeridad equivalente, $\mathrm{m} / \mathrm{s}$ & 846.32 & 1209.23 & 1578.30 \\
\hline Velocidad inicial, m/s & 0.66 & 0.45 & 0.37 \\
\hline Máxima sobrepresión, mca & 65.77 & 64.71 & 67.83 \\
\hline
\end{tabular}

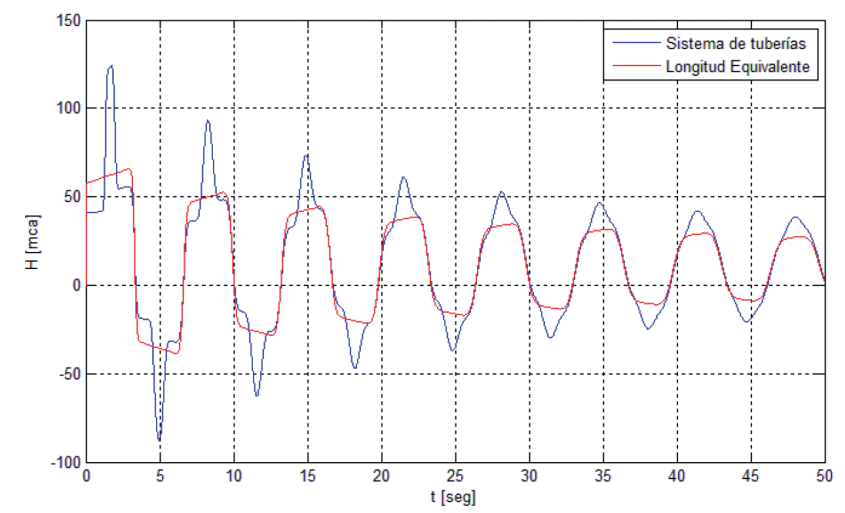

Figura 6: Variación de la sobrepresión en la válvula de control del sistema para ensanche $D_{1} / D_{2}=2 " / 4 "$ con ubicación de ensanche a una distancia $L_{1}=200 \mathrm{~m}$ 


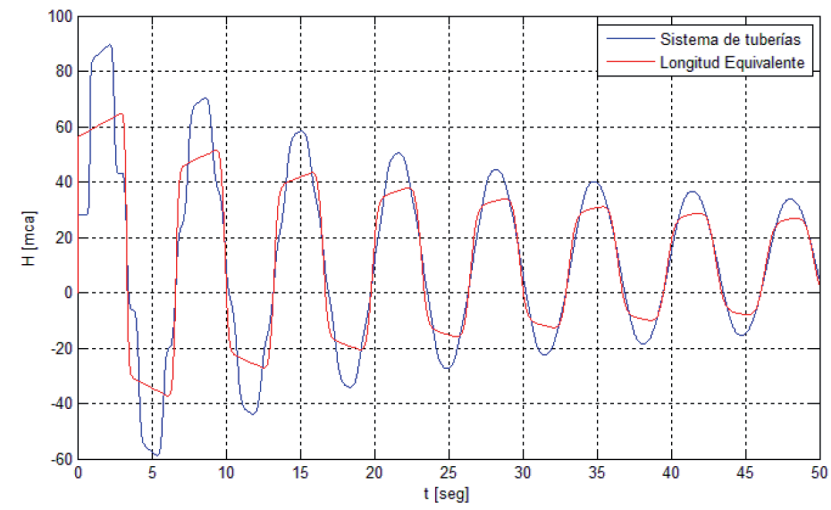

Figura 7: Variación de la sobrepresión en la válvula de control del sistema para ensanche $D_{1} / D_{2}=2 " / 4 "$ con ubicación de ensanche a una distancia $L_{1}=500 \mathrm{~m}$

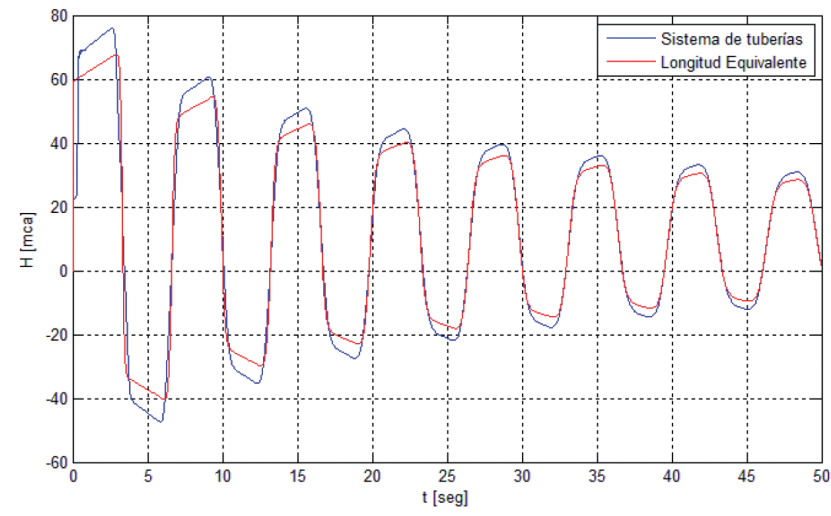

Figura 8: Variación de la sobrepresión en la válvula de control del sistema para ensanche $D_{1} / D_{2}=2 " / 4 "$ con ubicación de ensanche a una distancia $L_{1}=800 \mathrm{~m}$

\section{Caso angostamiento}

De igual manera se consideran dos casos de angostamientos, a saber: angostamiento de tubería de diámetro nominal 2.5 " a otra de 2" y angostamiento de diámetro nominal 2" a otra de 4". Para cada angostamiento considerado en la tubería se analizan tres casos, los cuales se diferencian por el lugar en el que se produce el cambio de diámetro. En el primer caso el angostamiento ocurre a $200 \mathrm{~m}$ del estanque, en el segundo caso a $500 \mathrm{~m}$ del estanque y el tercer caso a $800 \mathrm{~m}$ del estanque. En la Tabla 7 se muestran las características y resultados obtenidos de los tres sistemas que se analizan para el caso de angostamiento de 2.5 " a 2", mientras que en la Tabla 8 se indican las características y los resultados obtenidos del sistema aplicando el concepto de celeridad y longitud equivalente.

Las Figuras 9 a 14 muestran la comparación de los valores de la sobrepresión en función del tiempo en la válvula de control, obtenidos a través de la modelación numérica por elementos finitos y por el método de celeridad y longitud equivalente a distintas ubicaciones del cambio de geometría y para relaciones de cambio de angostamiento $D_{1} / D_{2}=2.5 " / 2 "$ y $D_{1} / D_{2}=4 " / 2 "$.

Tabla 7: Características definidas y valor de máxima sobrepresión obtenida para el caso de angostamiento de tubería de 2.5" a 2" a través de la modelación numérica por elementos finitos

\begin{tabular}{|l|c|c|c|c|c|c|}
\hline $\begin{array}{l}\text { Sistema de } \\
\text { tuberías }\end{array}$ & \multicolumn{2}{|c|}{ A } & \multicolumn{2}{c|}{ B } & \multicolumn{2}{c|}{ C } \\
\hline Tramo & 1 & 2 & 1 & 2 & 1 & 2 \\
\hline Longitud, $\mathrm{m}$ & 200 & 800 & 500 & 500 & 800 & 200 \\
\hline Diámetro, $\mathrm{m}$ & 0.058 & 0.049 & 0.058 & 0.049 & 0.058 & 0.049 \\
\hline Material & HDPE & HDPE & HDPE & HDPE & HDPE & HDPE \\
\hline Celeridad, m/s & 604.66 & 604.78 & 604.66 & 604.78 & 604.66 & 604.78 \\
\hline $\begin{array}{l}\text { Velocidad } \\
\text { inicial, } \mathrm{m} / \mathrm{s}\end{array}$ & 0.75 & 1.06 & 0.84 & 1.18 & 0.97 & 1.35 \\
\hline Caudal, $\mathrm{m} / \mathrm{s}$ & 0.0020 & 0.0022 & 0.0026 \\
\hline $\begin{array}{l}\text { Máxima } \\
\text { sobrepresión, } \\
\text { mca }\end{array}$ & \multicolumn{7}{|c|}{73.73} & \multicolumn{2}{|c|}{80.09} & \multicolumn{2}{c|}{90.21} \\
\hline
\end{tabular}

Tabla 8: Características definidas y valor de máxima sobrepresión obtenida para el caso de angostamiento de tubería de 2.5 " a 2 " a través del método de celeridad y longitud equivalente

\begin{tabular}{|l|c|c|c|}
\hline Sistema de tuberías & $\mathrm{A}$ & $\mathrm{B}$ & $\mathrm{C}$ \\
\hline Longitud equivalente, $\mathrm{m}$ & 1320 & 1200 & 1080 \\
\hline Diámetro equivalente, $\mathrm{m}$ & 0.058 & 0.058 & 0.058 \\
\hline Celeridad equivalente, m/s & 798.27 & 725.66 & 653.06 \\
\hline Velocidad inicial, m/s & 0.75 & 0.84 & 0.97 \\
\hline Máxima sobrepresión, mca & 70.19 & 71.03 & 73.18 \\
\hline
\end{tabular}

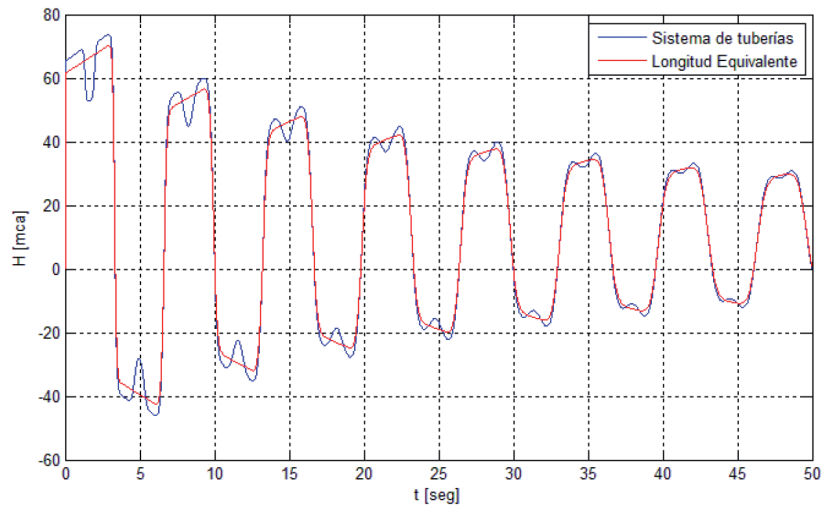

Figura 9: Variación de la sobrepresión en la válvula de control del sistema para angostamiento $D_{1} / D_{2}=2.5 \% / 2 "$ con ubicación de ensanche a una distancia $L_{1}=200 \mathrm{~m}$ 


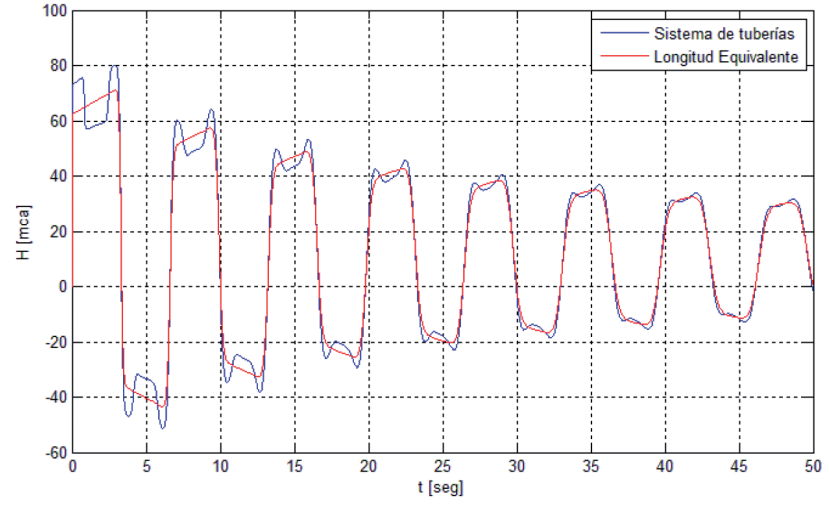

Figura 10: Variación de la sobrepresión en la válvula de control del sistema para angostamiento $D_{1} / D_{2}=2.5 \% / 2$ " con ubicación de ensanche a una distancia $L_{1}=500 \mathrm{~m}$

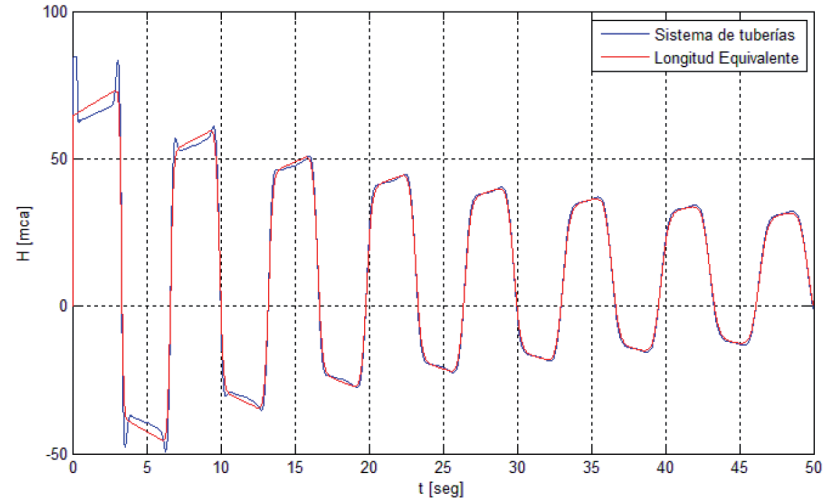

Figura 11: Variación de la sobrepresión en la válvula de control del sistema para angostamiento $D_{1} / D_{2}=2.5 \% / 2$ " con ubicación de ensanche a una distancia $L_{1}=800 \mathrm{~m}$

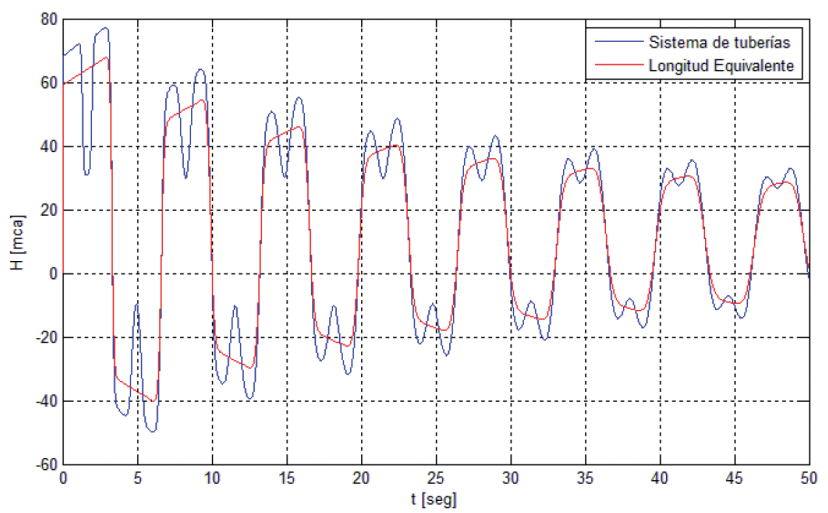

Figura 12: Variación de la sobrepresión en la válvula de control del sistema para angostamiento $D_{1} / D_{2}=4 " / 2$ " con ubicación de ensanche a una distancia $L_{1}=200 \mathrm{~m}$

En la Tabla 9 se muestran las características y resultados obtenidos de los tres sistemas que se analizan para el caso de angostamiento de 4" a 2", mientras que en la Tabla 10 se aprecian las características y los resultados obtenidos del sistema aplicando el concepto de celeridad y longitud equivalente.

Tabla 9: Características definidas y valor de máxima sobrepresión obtenida para el caso de angostamiento de tubería de 4" a 2" a través de la modelación numérica por elementos finitos

\begin{tabular}{|l|c|c|c|c|c|c|}
\hline $\begin{array}{l}\text { Sistema de } \\
\text { tuberías }\end{array}$ & \multicolumn{2}{|c|}{ A } & \multicolumn{2}{c|}{ B } & \multicolumn{2}{c|}{ C } \\
\hline Tramo & 1 & 2 & 1 & 2 & 1 & 2 \\
\hline Longitud, m & 200 & 800 & 500 & 500 & 800 & 200 \\
\hline Diámetro, m & 0.085 & 0.049 & 0.085 & 0.049 & 0.085 & 0.049 \\
\hline Material & HDPE & HDPE & HDPE & HDPE & HDPE & HDPE \\
\hline Celeridad, m/s & 604.45 & 604.78 & 604.45 & 604.78 & 604.45 & 604.78 \\
\hline $\begin{array}{l}\text { Velocidad } \\
\text { inicial, m/s }\end{array}$ & 0.37 & 1.10 & 0.45 & 1.36 & 0.66 & 1.98 \\
\hline Caudal, ms $/ \mathrm{s}$ & \multicolumn{2}{|c|}{0.0021} & 0.0026 & 0.0037 \\
\hline $\begin{array}{l}\text { Máxima } \\
\text { sobrepresión, } \\
\text { mca }\end{array}$ & \multicolumn{2}{|c|}{77.13} & \multicolumn{2}{|c|}{91.15} & 132.30 \\
\hline
\end{tabular}

Tabla 10: Características definidas y valor de máxima sobrepresión obtenida para el caso de angostamiento de tubería de 4" a 2" a través del método de celeridad y longitud equivalente

\begin{tabular}{|l|c|c|c|}
\hline Sistema de tuberías & $\mathrm{A}$ & $\mathrm{B}$ & $\mathrm{C}$ \\
\hline Longitud equivalente, $\mathrm{m}$ & 2610 & 2000 & 1400 \\
\hline Diámetro equivalente, $\mathrm{m}$ & 0.085 & 0.085 & 0.085 \\
\hline Celeridad equivalente, $\mathrm{m} / \mathrm{s}$ & 1578.30 & 1209.23 & 846.32 \\
\hline Velocidad inicial, m/s & 0.37 & 0.45 & 0.66 \\
\hline Máxima sobrepresión, mca & 67.77 & 64.64 & 65.61 \\
\hline
\end{tabular}

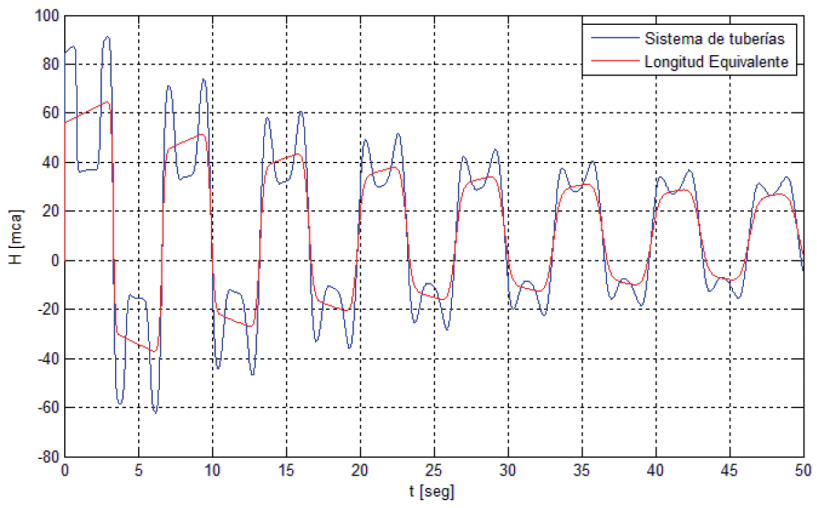

Figura 13: Variación de la sobrepresión en la válvula de control del sistema para angostamiento $D_{1} / D_{2}=4 " / 2 "$ con ubicación de ensanche a una distancia $L_{1}=500 \mathrm{~m}$ 


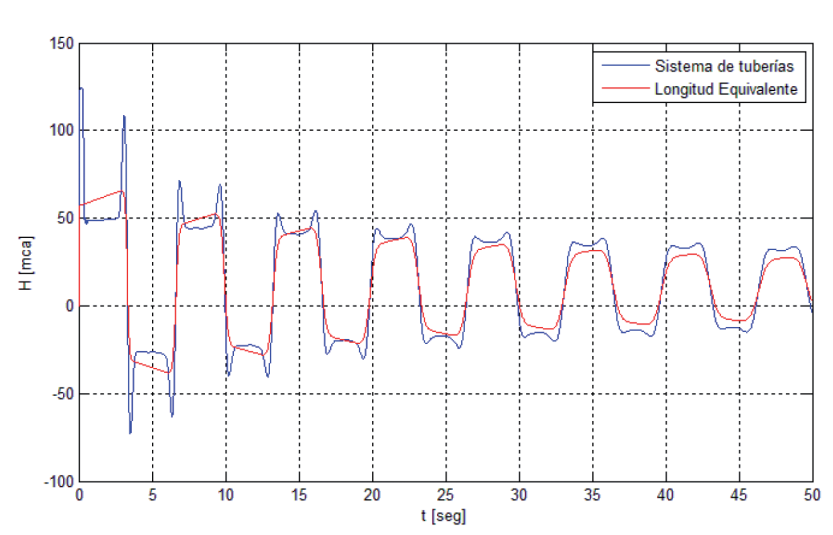

Figura 14: Variación de la sobrepresión en la válvula de control del sistema para angostamiento $D_{1} / D_{2}=4 " / 2$ " con ubicación de ensanche a una distancia $L_{1}=800 \mathrm{~m}$

\section{Conclusiones}

Se observa que tanto para el caso de ensanche de 2" a 2.5" $\left(D_{1} / D_{2}=2 " / 2.5 "\right)$ como para el caso de ensanche de $2 "$ a $4 "\left(D_{1} / D_{2}=2 " / 4 "\right)$ los resultados obtenidos a través de la modelación numérica por elementos finitos y por el método de celeridad y longitud equivalente son muy cercanos a partir de los $25 \mathrm{~s}$ de producirse el cierre (Figuras 3 a 5). Se aprecia que en los primeros $15 \mathrm{~s}$ existe una diferencia de magnitud en el valor de la máxima sobrepresión en la válvula. Esta diferencia de magnitud ocurre cuando el cambio de geometría se encuentra más cercano al estanque $\left(L_{1}=200 \mathrm{~m}\right)$. Se determina que la coincidencia de las ondas de presión obtenidas a través de la modelación numérica por elementos finitos y por el método de celeridad y longitud equivalente se debe a que la diferencia de celeridad entre un tramo y otro es mínima, debido a que el cambio de diámetro no ha sido muy significativo y las pérdidas singulares no juegan un rol determinante en este hecho, por lo que se mantiene una razón similar de diámetro y espesor. Sin embargo, este comportamiento no se refleja en los primeros segundos de análisis en donde se aprecia una diferencia marcada de sobrepresión por ambos métodos, siendo siempre mayor la obtenida a través de la modelación numérica por elementos finitos.

La situación es la misma cuando se analiza el caso de angostamiento, pero sólo para el caso de cambio de 2.5" a $2 "\left(D_{1} / D_{2}=2.5 " / 2 "\right)$. Cuando el angostamiento se hace más brusco, es decir, el caso de cambio de 4" a 2" ( $D_{1} /$ $\left.D_{2}=4 " / 2 "\right)$, los resultados entregados por ambos métodos presentan diferencia. Aquí los resultados obtenidos a través de la modelación numérica por elementos finitos y por el método de celeridad y longitud equivalente tienen la tendencia de acercarse a partir de los $25 \mathrm{~s}$ de producirse el cierre, pero sólo cuando dicho angostamiento se produce a una distancia $L_{1}=800 \mathrm{~m}$ (Figuras 12 a 14). Sin embargo, se aprecia que en los primeros $15 \mathrm{~s}$ la diferencia de magnitud en el valor de la máxima sobrepresión en la válvula se incrementa.

El método de celeridad y longitud equivalente no es capaz de representar los efectos de superposición y transmisión de ondas que ocurren en las discontinuidades de las tuberías debido a la variación de celeridad (variación de diámetro), entregando valores promedios de las ondas de presión que se desarrollan en cada tramo, lo que no permite simular correctamente lo que ocurre en el sistema de tuberías. Así la coincidencia del periodo de las ondas de presión obtenidos a través de la modelación numérica por elementos finitos y por el método de celeridad y longitud equivalente queda condicionada por la diferencia de celeridades entre un tramo y otro.

Una diferencia sustancial es que para los casos de ensanches la diferencia de presiones obtenidas a través de la modelación numérica por elementos finitos y por el método de celeridad y longitud equivalente es más evidente cuando el cambio de sección ocurre más lejos de la válvula, mientras que para los casos de angostamiento se produce cuando el cambio de sección ocurre más cerca de la válvula. Esto está relacionado con la transmisión y superposición de ondas que ocurre en la discontinuidad, y es más marcado para el caso del angostamiento dada la naturaleza experimental propia de estos cambios bruscos, efecto que el método de celeridad y longitud equivalente no es capaz de representar en sus resultados.

En términos generales la aproximación por el método de celeridad y longitud equivalente es capaz de representar las sobrepresiones generadas con un alto grado de exactitud. Sin embargo, la falencia de esta aproximación radica en que tiende a aminorar las máximas sobrepresiones, siendo éste el parámetro de mayor importancia en el análisis de movimentos transitorios en conductos a presión.

\section{Referencias}

Abreu, J.M., Guarga, R. e Izquierdo, J. (eds.) (1995). Transitorios y oscilaciones en sistemas hidráulicos a presión. Unidad Docente Mecánica de Fluidos, Universidad Politécnica de Valencia, España 
Allievi, L. (1903). Teoria generale del moto perturbato dell'acqua nei tubi in pressione (colpo d'ariete). Associazione Elettrotecnica Italiana, Roma, Italia

Bollrich, G. (2007). Technische Hydromechanik I. 6. Auflage. Huss-Medien GmbH, Berlin, Germany

Chung, J. and Hulbert, G.M. (1993). A time integration algorithm for structural dynamics with improved numerical dissipation: the generalized- $\alpha$ method. Journal of Applied Mechanics 60(2), 371-375

Hughes, T.J.R. (1987). The finite element method: linear static and dynamics finite element analysis. Prentice-Hall, New Jersey, USA

Jansen, K.E., Whiting, C.H. and Hulbert, G.M. (2000). A generalized- $\alpha$ method for integrating the filtered Navier-Stokes equations with a stabilized finite element method. Computer Methods in Applied Mechanics and Engineering 190(3-4), 305319

Kong, A. y Salazar, I. (2017). Sobre la influencia de la heterogeneidad de material en el cálculo de la sobrepresión en ductos en presión. Ingeniería Innova 13, 1-16
Parmakian, J. (1963). Waterhammer analysis. Dover Publications, New York, USA

Salazar, I., Köngeter, J. y Covarrubias, A. (2011). El método de los elementos finitos aplicados a problemas de mecánica de fluidos. Revista de Ingeniería Innova 1, 18-27

Streeter, V.L. y Wylie, E.B. (1999). Mecánica de fluidos. $8^{\mathrm{a}}$ ed., McGraw-Hill, México

Twyman, J. (2019). Análisis transiente de la demanda de agua uniformemente distribuida. Obras y Proyectos 26, 43-50

Twyman, J. (2018). Transient flow analysis using the method of characteristics MOC with five-point interpolation scheme. Obras y Proyectos 24, 62-70

Twyman, J. (2016). Wave speed calculation for water hammer analysis. Obras y Proyectos 20, 86-92

Videla, D. y Salazar, I. (2010). Comparación del método de Crank Nicholson y $\alpha$-generalizado aplicados a la ecuación de flujo de aguas subterráneas. Obras y Proyectos 7, 51-56

Zienkiewicz, O. y Taylor, R. (2000). El método de los elementos finitos. $5^{\mathrm{a}}$ edición CIMNE, Barcelona, España 